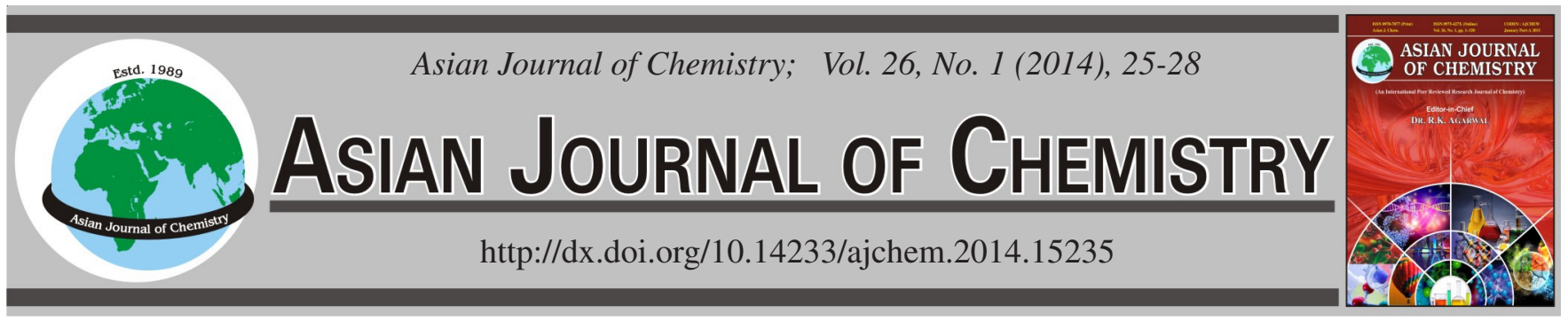

\title{
Morphology and Properties of Polypeptide-block-poly(ethylene glycol)/ Poly(tetramethylene glycol) Blend Film
}

\author{
Guo-Quan Zhu, Fa-Gang Wang ${ }^{*}$, Chang-Hong Su, Qiao-Chun GaO and Yu-Ying Liu
}

School of Materials Science and Engineering, Shandong University of Technology, Zibo 255049, P.R. China

*Corresponding author: E-mail: fagangwang@126.com

\begin{abstract}
A series of poly( $\gamma$-benzyl L-glutamate)-block-poly(ethylene glycol)/poly(tetramethylene glycol) (PBLG-block-PEG/PTMG) blend films were prepared by casting the polymer blend solution in dichloroethane. Surface morphologies of the PBLG-block-PEG/PTMG blend films were investigated by scanning electron microscopy and atomic force microscopy. Mechanical and chemical properties of the polymer blend films were studied by tensile tests and surface contact angle tests. It was shown that the introduction of poly(tetramethylene glycol) could greatly affect the properties of the polypeptide block copolymer films.
\end{abstract}

Keywords: Polypeptide block copolymer, Blend film, Morphology.

\section{INTRODUCTION}

In recent years, the polymer blending has been a highly useful method for the improvement or modification of physicochemical properties of the polymer materials ${ }^{1,2}$. An important property of the polymer blend is the miscibility of its ingredients, as it affects the mechanical properties, morphology, permeability and degradation ${ }^{1,2}$. Many studies regarding the miscibility in multi-component polymer systems have been reported. Among them, the polymer blends between biopolymers and synthetic polymers are of particular significance as they could be used as biomedical and biodegradable materials ${ }^{3-7}$.

Based on the excellent biocompatibility and biodegradability, the polypeptides and their copolymers have received attention for their great potential applications ${ }^{8-14}$. The polypeptides and their copolymers have been investigated widely in the fields of functional biomaterials, protein simulation, polymer carriers for protein conjugates, macromolecular conformational study, catalysis, nanoreactors and drug delivery systems, etc. ${ }^{15-21}$. For the polypeptide copolymer films, an important application is for temporary artificial skin substrates in burn therapy ${ }^{8,22}$.

Compared with pure polypeptide copolymers, polypeptide copolymers/synthetic polymer blends have received relatively little attention. As known, poly(tetramethylene glycol) (PTMG) holds good flexibility, weak hydrophilicity and better biocompatibility and biodegradability ${ }^{23,24}$, the introduction of PTMG chains could modify the properties of the polypeptide block copolymer film and increase its research fields. However, to the best of our knowledge, no experimental work has been reported on the properties of polypeptide block copolymer/PTMG blend films so far. In this work, PBLGblock-PEG/PTMG blend films were prepared by casting the polymer blend solution in dichloroethane. Surface morphologies of the polymer blend films were investigated by atomic force microscopy (AFM) and scanning electron microscopy (SEM). Mechanical and chemical properties of the polymer blend films were studied by tensile tests and surface contact angle tests. It was revealed that the introduction of PTMG could marked affect the properties of the polypeptide block copolymer films.

\section{EXPERIMENTAL}

The poly(tetramethylene glycol) (PTMG) $(\mathrm{Mw}=2000)$ was purchased from Dongda Chemical Co. Ltd. (China). Amine-terminated $\alpha$-methoxy- $\omega$-amino poly(ethylene glycol) (AT-PEG, $M_{w}=5000$ ) was purchased from Sigma Inc. (USA) and used without further purification. Hexane, tetrahydrofuran (THF) and 1,4-dioxane are of analytical grade and dried with sodium to remove water before use. Dichloroethane and other solvents are of analytical grade and used without further purification.

Synthesis of polypeptide block copolymer: PBLGblock-PEG copolymer was prepared by a standard $N$-carboxyl$\gamma$-benzyl- $L$-glutamate anhydride (NCA) method ${ }^{17,25}$. Briefly, PBLG-block-PEG copolymer was obtained by the ring-opening polymerization of $\gamma$-BLG NCA initiated by AT-PEG $\left(\mathrm{M}_{\mathrm{w}}=\right.$ 
5000 ) in 1,4-dioxane at room temperature. The reaction mixture was poured into a large volume of anhydrous methanol. The precipitated product was dried under vacuum and then purified twice by repeated precipitation from a chloroform solution into a large volume of anhydrous methanol. The molecular weight of PBLG-block-PEG copolymer was estimated by NMR measurements ${ }^{17}$. The molecular weight of PBLG-blockPEG used in the study was about 55000.

Preparation of PBLG-block-PEG/PTMG blend film: The polymer blend films were prepared by casting a $35 \mathrm{wt} \%$ polymer blend solution in dichloroethane onto clean glass plates and drying them under vacuum at $60{ }^{\circ} \mathrm{C}$. Also, it is found that, when PTMG mole content in polymer blend is over $15 \%$, the polymer blend can not form an even and continuous film.

Test method: SEM investigation was carried out using a scanning electron microscope (Sirin 200, FEI, Holland). Gold was sprayed on the polymer blend films in vacuum. Acceleration voltage was $10 \mathrm{kV}$. AFM investigation was carried out on a NanoScope IIIA MultiMode AFM instrument (Digital Instruments Inc., USA) in air at ambient conditions using tapping mode probes with constant amplitude $(200 \mathrm{mV})$. The rotated tapping mode etched silicone probe with a resonance frequency of $250 \mathrm{kHz}$ was used ${ }^{10}$. Tensile tests were carried out with an Instron Model 4468 universal testing machine (Digital Instruments, USA). The crosshead speed was set to $80 \mathrm{~mm} / \mathrm{min}$. For each data point, five samples were tested and the average value was taken. A $5 \mu \mathrm{L}$ drop of pure distilled water was placed on the polymer blend film surface using a syringe with a 22-gauge needle. The static contact angle was measured with an optical contact angle meter CAM 200 (KSV Instrument Ltd., Finland). The measurements of each contact angle were performed within $10 \mathrm{~s}$ after each drop to ensure that the droplet did not soak into the compact. The surface contact angles were the mean of five determinations ${ }^{10}$. Water-absorption ratio measurements of the polymer blend films were carried out as follows: at room temperature, the sample films were soaked in distilled water for $12 \mathrm{~h}$ and then filter paper was used to wipe off water from the blend film surface. The water-absorption ratio (\%) was calculated according to the formula: Water-absorption ratio $(\%)=\left[\left(\mathrm{m}_{2}-\mathrm{m}_{1}\right) / \mathrm{m}_{1}\right] \times 100 \%$, where $\mathrm{m}_{1}$ and $\mathrm{m}_{2}$ are the masses of the polymer blend films before and after being immersed in distilled water, respectively ${ }^{14}$

\section{RESULTS AND DISCUSSION}

The surface morphologies of PBLG-block-PEG/PTMG blend films with various PTMG mole contents were studied by SEM technique. Fig. 1 shows the surface morphologies of the polymer blend films with various PTMG mole contents: (a) 0 and (b) $15 \%$. As can be seen from Fig. 1, the surface of the polypeptide block copolymer film is relatively uniform, while the surface of the polymer blend film is coarse and inhomogeneous, which is caused by the interaction between the polypeptide block copolymer chains and the PTMG segments by entanglement. This phenomenon suggests that the difference of the surface morphologies between the polypeptide block copolymer film and the polymer blend film could be attributed to the introduction of PTMG segments.
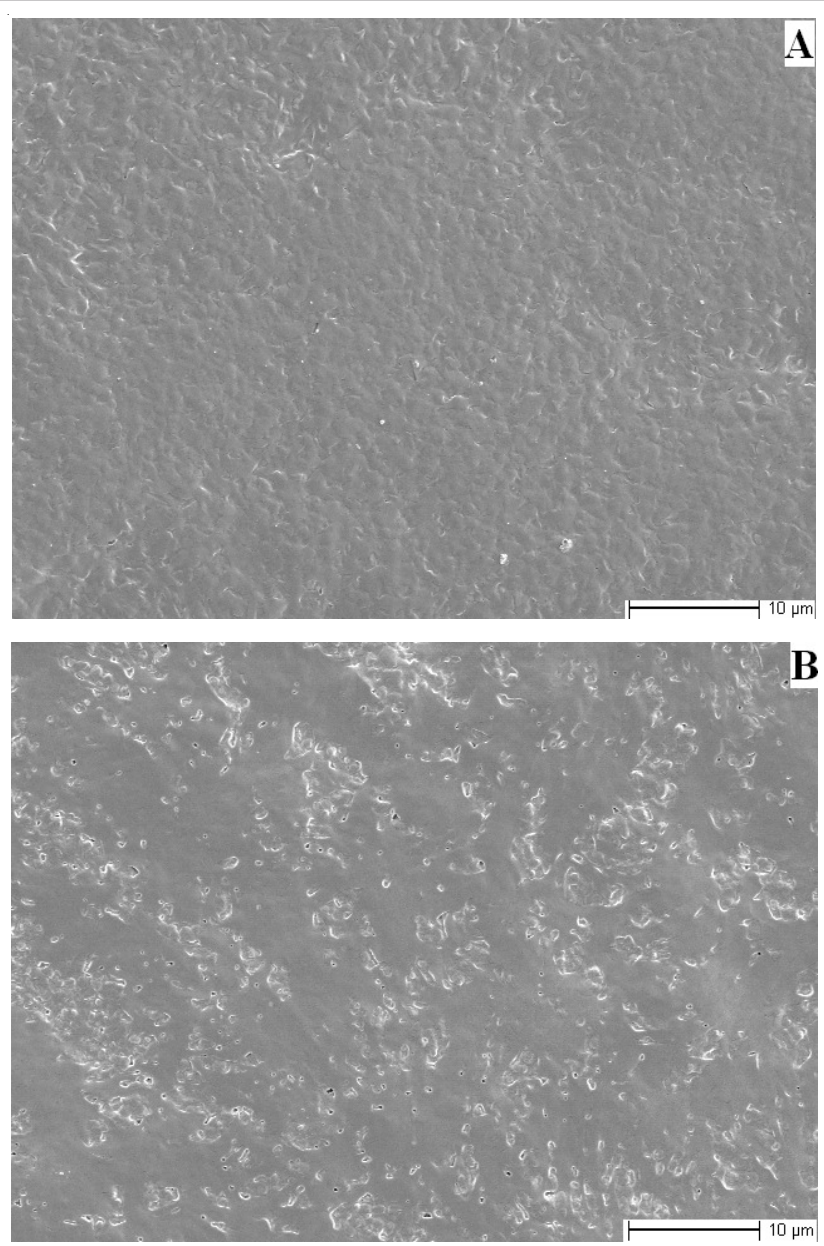

Fig. 1. SEM photographs of the PBLG-block-PEG/PTMG blend film surface with various PTMG mole contents: (a) 0 and (b) $15 \%$

Atomic force microscopic tests: As known, the AFM height images indicated the surface roughness of the polymer film $^{26-28}$. Fig. 2 presents the AFM 3D height images of PBLGblock-PEG/PTMG blend film surface with various PTMG mole contents: (a) 0 and (b) $15 \%$. As seen from Fig. 2, the surface colors of the polypeptide block copolymer film and the polymer blend film all take on both brighter domains (corresponding to the higher part) and darker domains (corresponding to the lower part) and the ratio of brighter domains to darker domains is different, indicating the surface roughness of the polypeptide block copolymer film is changing with the introduction of the PTMG segments ${ }^{26-28}$. This phenomenon reveals that the surface roughness difference of the polypeptide block copolymer film and the polymer blend film could also be attributed to the introduction of the PTMG segments.

Tensile tests: Fig. 3 displays the relationship between the tensile strength of the polymer blend film and the PTMG mole contents. As is shown in Fig. 3, the tensile strength of the polymer blend film decreased with the increase of the PTMG mole contents in the polymer blend. As mentioned above, compared with the polypeptide block copolymer, PTMG is very flexible, suggesting the decrease of the tensile strength of polymer blend film is related with the introduction of the flexible PTMG segments. Under permitted PTMG mole content, the higher the PTMG mole content, the lower the tensile strength of the polymer blend film. 


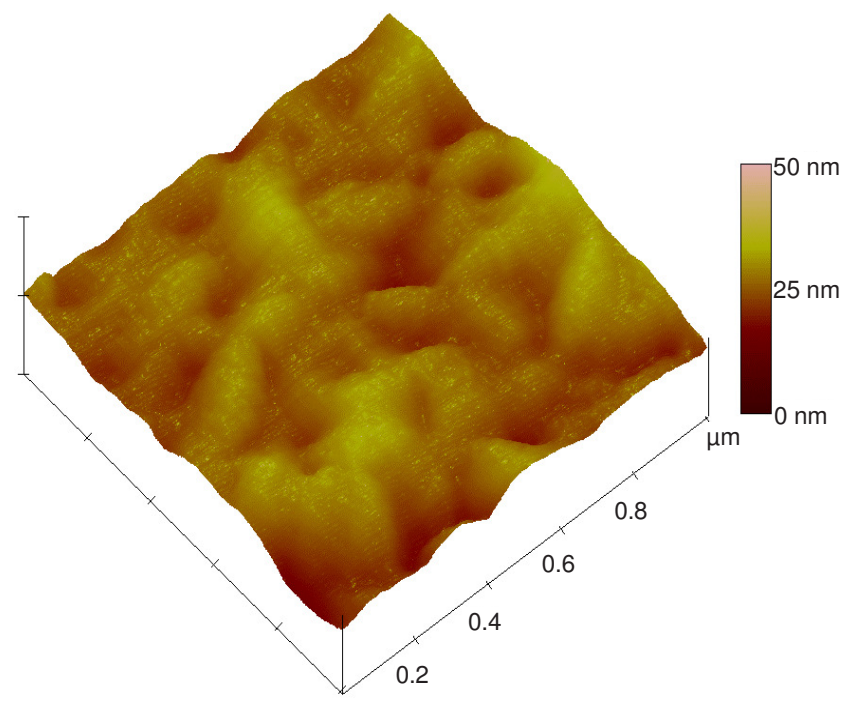

(a)

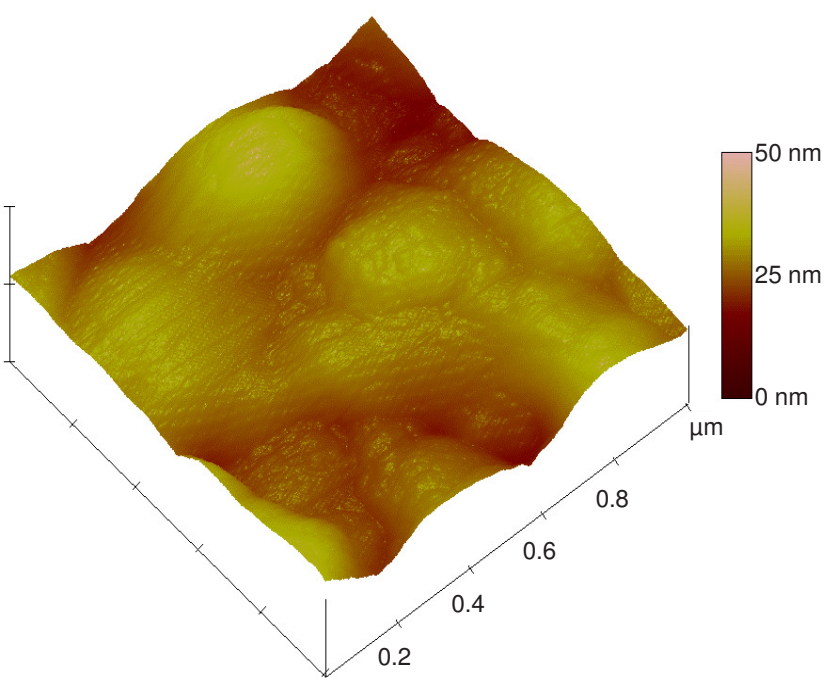

(b)

Fig. 2. AFM 3D height images of the PBLG-block-PEG/PTMG blend film surface with different PTMG mole contents: (a) 0 and (b) $15 \%$

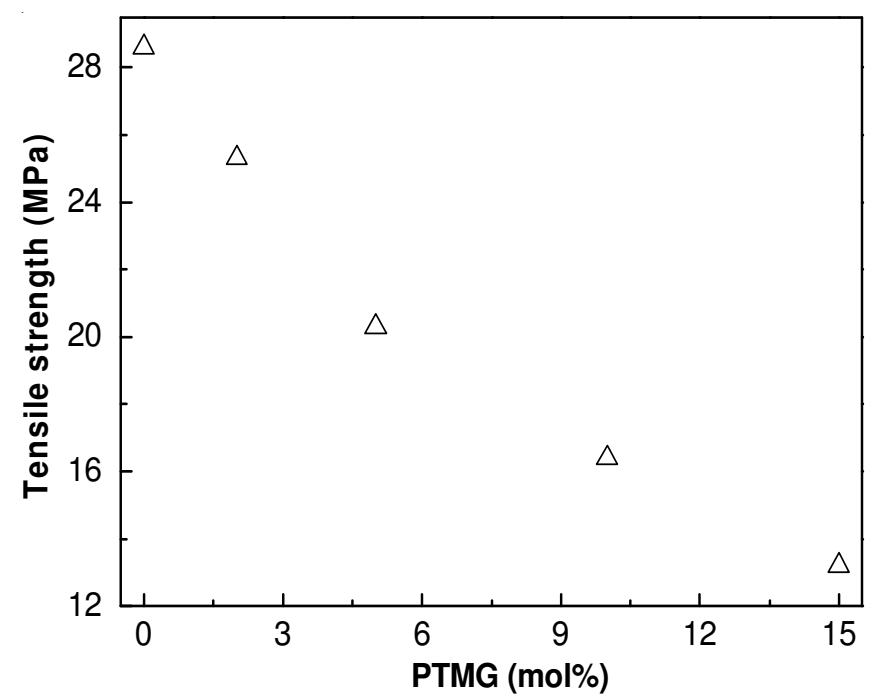

Fig. 3. Relationship between the tensile strength of the polymer blend film and the PTMG mole contents
Surface contact angle tests: Fig. 4 indicates the relationship between the surface contact angle of the polymer blend film and the PTMG mole contents. As shown in Fig. 4, the surface contact angle of the polymer blend film slightly decreased with increasing the PTMG mole contents in the polymer blend, indicating that the hydrophilicity of the polymer blend film slightly increased. As known, PBLG segments are hydrophobic and PEG chains hold hydrophilicity, suggesting the polypeptide block copolymer (wt ratio of PBLG segments to PEG segments is 10:1) holds a little hydrophilicity. Compared with hydrophobic PBLG segments, PTMG chains hold a little hydrophilicity, suggesting the introduction of the PTMG chains slightly increased the hydrophilicity of the polypeptide block copolymer film. This phenomenon proves that the decrease of the surface contact angle of the polymer blend film was concerned with the introduction of the PTMG chains.

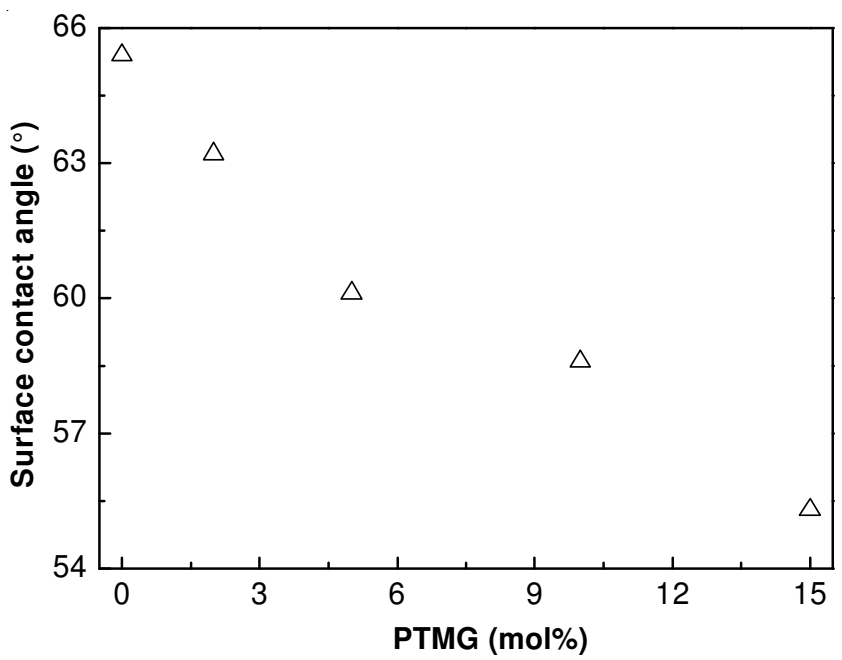

Fig. 4. Relationship between the surface contact angle of the polymer blend film and the PTMG mole contents

Water-absorption ratio tests: Fig. 5 represents the relationship between the water-absorption ratio of the polymer blend film and the PTMG mole contents. As it can be seen from Fig. 5, the water-absorption ratio of the polymer blend film increased with the increase of the PTMG mole content. As discussed above, the introduction of the PTMG chains increased the hydrophilicity of the polypeptide block copolymer film, suggesting the increase of the water-absorption ratio of the polymer blend film was also related with the introduction of the PTMG chains.

\section{Conclusion}

A series of PBLG-block-PEG/PTMG blend films were prepared by casting the polymer blend solution in dichloroethane. Surface morphologies of the polymer blend films were investigated by SEM and AFM. Mechanical and chemical properties of the polymer blend films were studied by tensile tests and surface contact angle tests. SEM photographs attested that the introduction of the PTMG chains changed the surface morphologies of the polypeptide block copolymer film. AFM images showed that the introduction of the PTMG segments also changed the surface roughness of the polypeptide block copolymer film. Tensile tests verified that the introduction of the PTMG segments decreased the tensile strength of the 


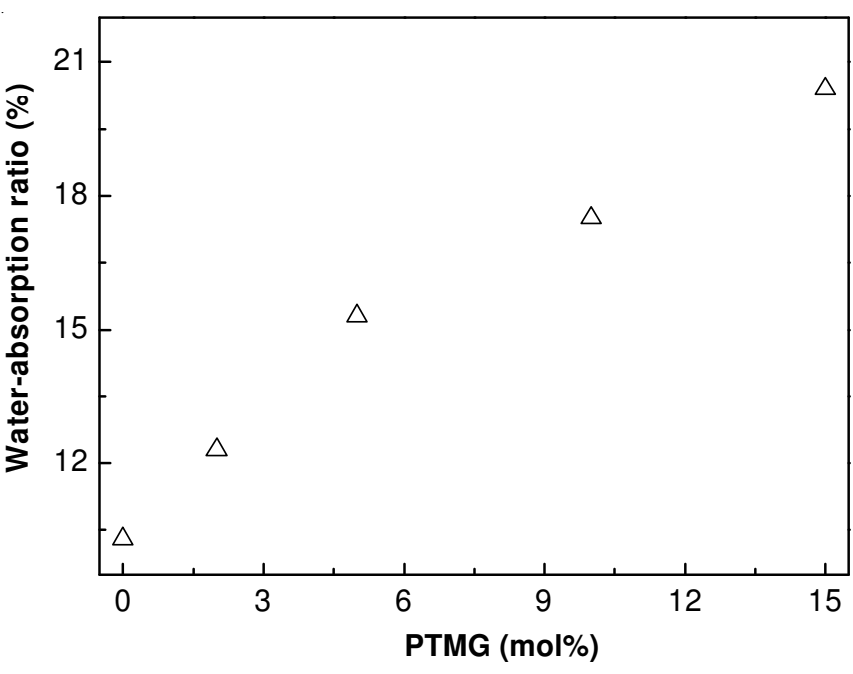

Fig. 5. Relationship between the water-absorption ratio of the polymer blend film and the PTMG mole contents

polypeptide block copolymer film. Both the surface contact angle tests and the water-absorption ratio tests proved that the introduction of the PTMG chains increased the hydrophilicity of the polypeptide block copolymer film.

\section{ACKNOWLEDGEMENTS}

This work is supported by the Natural Science Foundation of Shandong Province (No. ZR2011EMM009).

\section{REFERENCES}

1. Y. Nishio and R. Manley, Macromolecules, 21, 1270 (1988).

2. J.S. Park, J.W. Park and E. Ruckenstein, Polymer, 42, 4271 (2001).

3. T. Kondo, C. Sawatari, R. Manley and D.G. Gray, Macromolecules, 27, 210 (1994).

4. C. Sawatari and T. Kondo, Macromolecules, 32, 1949 (1999).
5. K. Lio, N. Minoura and M. Nagura, Polymer, 36, 2579 (1995).

6. G.Q. Zhu, G.C. Li and P. Wang, Polym.-Plast. Technol. Eng., 50, 1470 (2011).

7. G.Q. Zhu, Y.Y. Liu and F.G. Wang, Int. J. Polym. Mater., 61, 737 (2012).

8. K. Jokei, M. Oka, T. Hayashi and Y. Miyachi, Eur. Polym. J., 35, 945 (1999).

9. D.M. Tang, J.P. Lin, S.L. Lin, S.N. Zhang, T. Chen and X.H. Tian, Macromol. Rapid Commun., 25, 1241 (2004).

10. L.Q. Bai, L.J. Zhu, S.J. Min, L. Liu, Y.R. Cai and J.M. Yao, Appl. Surf. Sci., 254, 2988 (2008).

11. I. Oh, K. Lee, H.-Y. Kwon, Y.-B. Lee, S.-C. Shin, C.-S. Cho and C.-K. Kim, Int. J. Pharm., 181, 107 (1999).

12. G.Q. Zhu, L. Feng and S.N. Zhang, J. Macromol. Sci. A, 46, 694 (2009).

13. N. Higashi, J. Kawahara and M. Niwa, J. Colloid Interf. Sci., 288, 83 (2005).

14. G.Q. Zhu, Chem. Pap., 64, 34 (2010).

15. Z. Gao, A. Desjardins and A. Eisenberg, Macromolecules, 25, 1300 (1992).

16. X.F. Zhong, S.K. Varshney and A. Eisenberg, Macromolecules, 25, 7160 (1992).

17. T. Li, J.P. Lin, T. Chen and S.N. Zhang, Polymer, 47, 4485 (2006)

18. J.P. Lin, A. Abe, H. Furuya and S. Okamoto, Macromolecules, 29, 2584 (1996).

19. G.Q. Zhu, F.G. Wang, Y.Y. Liu and Q.C. Gao, Fibers Polym., 11, 819 (2010).

20. J.P. Lin, G.Q. Zhu, X.M. Zhu, S.L. Lin, T. Nose and W.W. Ding, Polymer, 49, 1132 (2008).

21. M. Moffitt and A. Eisenberg, Macromolecules, 30, 4363 (1997).

22. Y. Miyachi, K. Jokei, M. Oka and T. Hayashi, Eur. Polym. J., 35, 767 (1999).

23. H.-Y. Tsi, C.-C. Chen, W.-C. Tsen, Y.-C. Shu and F.-S. Chuang, Polym. Test., 30, 50 (2011)

24. S.C. Roh, M.J. Park, S.H. Yoo and C.K. Kim, J. Membr. Sci., 411, 201 (2012).

25. G.Q. Zhu, Q.C. Gao, Z.H. Li and F.G. Wang, Int. J. Polym. Mater., 60, 290 (2011); .

26. M.Y. Lee, S.H. Kim, H.S. Ganapathy, S.W. Kim and K.T. Lim, Ultramicroscopy, 108, 1210 (2008).

27. M.J. Fasolka, A.M. Mayes and S.N. Magonov, Ultramicroscopy, 90, 21 (2001).

28. S. Yamamoto, Y. Tsujii and T. Fukuda, Polymer, 42, 2007 (2001). 